\title{
Stability Control in A Binary Fluid Mixture Subjected to Cross Diffusive Coefficients
}

\author{
Nurul Hafizah Zainal Abidin ${ }^{\# 1}$, Nor Fadzillah Mohd Mokhtar ${ }^{\# 2}$, Izzati Khalidah Khalid ${ }^{\# 3}$, Rosleela Abdul \\ Rahim $^{\#}$, Siti Salwa Abd Gani ${ }^{+}$ \\ ${ }^{\#}$ Institute for Mathematical Research, Universiti Putra Malaysia, 43400 Serdang, Selangor, Malaysia \\ E-mail: ${ }^{1}$ nurul354@perak.uitm.edu.my, ${ }^{2}$ norfadzillah.mokhtar@gmail.com, ${ }^{3}$ izzatikk@yahoo.com, ${ }^{4}$ rossz022593@gmail.com \\ ${ }^{\#}$ Department of Mathematics, Faculty of Science, Universiti Putra Malaysia, 43400 Serdang, Selangor, Malaysia \\ ${ }^{+}$Department of Chemistry, Faculty of Science, Universiti Putra Malaysia, 43400 Serdang, Selangor, Malaysia \\ ssalwaag@upm.edu.my \\ ${ }^{\#}$ Faculty Science Computer and Mathematical Sciences, Universiti Teknologi MARA, 35400 Tapah, Perak, Malaysia
}

\begin{abstract}
The effect of feedback control on the onset of double-diffusive convection in a horizontal binary fluid layer is studied analytically subjected to cross diffusion coefficients which are the Soret and Dufour parameters. The confined boundaries of the binary fluid layer are considered to be free-free, rigid-free and rigid-rigid which described the lower and upper surfaces respectively. The linear stability theory is applied, and the eigenvalue solution is obtained numerically using Galerkin technique. Focusing on the stationary convection, it is shown that there is a positive thermal resistance in the presence of feedback control on the onset of doublediffusive convection in binary fluid mixture.
\end{abstract}

Keywords — feedback control; binary fluid; Soret parameter; Dufour

\section{INTRODUCTION}

In the past few decades, great observations of doublediffusive convection have been rapidly investigated by many scientists because of their applications in various physical phenomena, such as oceanography, crystal production, metallurgy, and astrophysics. Double-diffusive convection is a process of interaction of two fluid components that diffuse at imbalance rates. In thermosolutal convection, buoyancy forces can arise not only from density differences due to variation in temperature gradient but also from those due to variation cross diffusive coefficients viz., Soret and Dufour effects. In [1] studied oceanographical phenomena where they found that the ascending (or descending) water in the tube would exchange heat but not salinity with the ambient ocean and would be accelerated due to its deficit in salt and density relative to fluid at the same level outside the tube. In [2] investigated the thermohaline convection and discussed the stability characteristics in laminar flow. In [3] analyzed the effect of the thermal and solutal gradient in thermosolutal convective instability in a double-diffusive fluid layer where both effects either can stabilize or destabilize the stationary and oscillatory mode. In [4] demonstrated the soret-driven thermosolutal convection both theoretically and experimentally using a water-methanol mixture. Then, in [5] continue previous investigation using water-ethanol mixture while [6] extended the investigation using salt solution. In [7] reviewed the study on double-diffusive convection. In [8] studied the convection in liquids. The linear stability of experimental Soret convection in water-ethanol mixture with various boundary conditions has been investigated by [9] with an emphasized on Biot number. The onset of convection in binary mixtures with Dufour effect was investigated by [10]. It is shown that Dufour parameter can have a significant influence on the stability boundaries of the quiescent state depending on the strength of the Dufour coupling. The thermocapillary instability in the binary fluid on the onset of convection by considering the Soret effect with other physical influences has been studied by [11], [12] followed by [13]. In 2011, in [14] use the linear instability theory to study the onset of double-diffusive convection in a horizontal layer of nanofluids heated and salted from below. Double diffusive convection of fluid with Soret and Dufour effects are studied by [15]. The expressions for both stationary and oscillatory Rayleigh numbers which characterized the stability of the system are obtained. They 
found that in the presence of cross-diffusive in system heated and salted from above, the oscillatory convection has been delayed at the marginal state. The scientists and researchers have started the investigation on delaying the onset of convective heat transfer through the use the controller strategy. In [16], [17] worked on actively controlled convection in the Rayleigh-Benard system and concluded that the controller gain stabilized a non-stable state. In [18], [19] used a controller that consists of sensors that detect deviations in the temperature of the fluid and showed the similar control strategy could be used to control the flow patterns of the system. These series of papers by [20]-[22] analyzed the active control through the uses of shadow graphic measurement on the onset of Rayleigh-Benard convection and found that the simple linear control stabilized the unstable no-motion state. In [23], [24] experimented the use of feedback control and pointed out that without correct temperature, power control, saturation of actuators, conduction through the side wall and an insufficient number of actuators can lead the controller to achieve bad performance. In [25] demonstrated the critical Marangoni number could be increased through the use of feedback control. In [26] found the onset of Marangoni-Benard convection with internal heat generation can be delayed through the use of feedback control. In [27], [28] found that an active control strategy significantly effective in suppressing the onset of Marangoni convection. In [29] demonstrated the critical Marangoni number could be drastically increased with the combination effects of feedback control and rotation. In [30] found the onset of surface tension instability can be delayed through the use of feedback control in Eringen fluid. In [31] applied the effect of feedback control on the onset of Rayleigh-Benard convection in micropolar fluid and found that it has a stabilizing factor to the system.

The study of feedback control on the onset of convection in the binary fluid mixture has not been given any attention in spite of its importance in controlling the stability. To fill the void, this present analysis attempts to study the effect of feedback control in the presence of Dufour and Soret effects on the onset of the double-diffusive binary fluid layer. Three types of bounding surfaces (lower boundary-upper boundary) are considered in this investigation: free-free, rigid-free and rigid-rigid. We assume that the upper surface to be non-deformable and employed the stability analysis theory. The resulting eigenvalue problem is solved using the Galerkin method.

\section{MATERIAL AND METHOD}

Consider an infinite horizontal layer of binary fluid of depth, $d$ confined between the planes $z=0$ and $z=d$ subjected to the feedback control, $K$ is heated from below. The stability of a horizontal layer of binary fluid in the presence of feedback control is examined. We select a Cartesian coordinate system with $z$ pointing upward, opposite to the gravity vector, and $(x, y)$ in the horizontal direction at the rigid bottom boundary. The onset of doublediffusive convection is studied under the Boussinesq approximation where the density $(\rho)$ is assumed to be linearly dependent upon the temperature $(T)$ and the solute concentration $(C)$ which is given by

$$
\rho=\rho_{0}\left[1-\alpha\left(T-T_{0}\right)+\alpha_{c}\left(C-C_{0}\right)\right]
$$

Here, $\alpha=-\rho_{0}^{-1} \partial \rho / \partial T$ and $\alpha_{c}=-\rho_{0}^{-1} \partial \rho / \partial C$. Assuming lower boundary at a higher temperature than the upper boundary and these boundaries are maintained at a constant temperature. Let the solute concentrations to be taken as $C_{0}$ $+\Delta C$ and $C_{0}$. Following analysis by [4] for Soret parameter and [10] for Dufour effect, the governing equations for the Rayleigh-Benard convection under the Oberbeck-Boussinesq approximation are given as

$$
\begin{gathered}
\nabla \cdot v=0, \\
\rho_{0}\left[\frac{\partial v}{\partial t}+(v \cdot \nabla) v\right]=-\nabla p+\mu \nabla^{2} v-\rho g e_{z}, \\
{\left[\frac{\partial T}{\partial t}+(v \cdot \nabla) T\right]=\kappa \nabla^{2} T+D_{T C} \nabla^{2} C,} \\
{\left[\frac{\partial C}{\partial t}+(v \cdot \nabla) C\right]=\kappa_{c} \nabla^{2} C+D_{C T} \nabla^{2} T .}
\end{gathered}
$$

The basic state of the fluid is quiescent and is given by

$$
\begin{gathered}
(u, v, w)=(0,0,0), T=T_{b}(z), p=p_{b}(z), \rho=\rho_{b}(z) \text { and } \\
C=C_{b}(z)
\end{gathered}
$$

On the basic state, we superpose perturbations in the form

$$
\begin{gathered}
(u, v, w, T, p, \rho, C)=\left[0,0,0, T_{b}(z), p_{b}(z), \rho_{b}(z), C_{b}(z)\right] \\
{\left[u^{\prime}, v^{\prime}, w^{\prime}, T^{\prime}, p^{\prime}, \rho^{\prime}, C^{\prime}\right]}
\end{gathered}
$$

where the primes quantities indicate the perturbed variables.

The Equation (2)-(5) are non-dimensionalized using the following definitions

$$
\begin{aligned}
& \left(x^{\prime}, y^{\prime}, z^{\prime}\right)=\frac{(x, y, z)}{d}, \\
& t^{\prime}=\frac{t \kappa}{d^{2}}, \\
& \left(u^{\prime}, v^{\prime}, w^{\prime}\right)=\frac{d(u, v, w)}{\kappa}, \\
& p^{\prime}=\frac{p d^{2}}{v \kappa \rho}, \\
& T^{\prime}=\Delta T, \\
& C^{\prime}=\Delta C,
\end{aligned}
$$

and using Equation (6) and Equation (7), we obtain the nondimensional variables

$$
\begin{aligned}
& \nabla \cdot v^{\prime}=0 \\
& \frac{1}{\operatorname{Pr}} \frac{\partial v^{\prime}}{\partial t}=-\nabla p^{\prime}+\nabla^{2} v^{\prime}+R a T^{\prime} \hat{e}_{z}+\left(\frac{R s}{L e}\right) C^{\prime} \hat{e}_{z}, \\
& \frac{\partial T^{\prime}}{\partial t}+w^{\prime}=\nabla^{2} T^{\prime}+D f \nabla^{2} C^{\prime}, \\
& \frac{\partial C^{\prime}}{\partial t}-w^{\prime}=\frac{1}{L e} \nabla^{2} C^{\prime}+S r \nabla^{2} T^{\prime},
\end{aligned}
$$

where 


$$
\begin{aligned}
& R a=\frac{\alpha g d^{3} \nabla T}{v \kappa}, \\
& R s=\frac{\alpha_{c} g d^{3} \nabla C}{v \kappa_{c}}, \\
& L e=\frac{\kappa}{\kappa_{c}}, \\
& D f=\frac{D_{T C} \Delta T}{\kappa \Delta T}, \\
& S r=\frac{D_{C T} \Delta T}{\kappa \Delta C}, \\
& \operatorname{Pr}=\frac{v}{\kappa} .
\end{aligned}
$$

Operating on Equation (10) by eliminating the pressure term by using curl identity together with Equation (9), Equation (10) can be written as

$$
\left[\frac{1}{\operatorname{Pr}} \frac{\partial}{\partial t} \nabla^{2}-\nabla^{4}\right] w^{\prime}=R a T^{\prime} \nabla^{2}+\left(\frac{R s}{L e}\right) C^{\prime} \nabla^{2} .
$$

A normal mode representation is introduced in the form

$$
\left(w^{\prime}, T^{\prime}, C^{\prime}\right)=[W(z), \theta(z), \xi(z)] e^{\left[i\left(a_{x}^{x}+a_{y}^{y}+\sigma t\right)\right]} .
$$

Substituting Equation (15) into Equation (14) and Equation (11)-(12) we obtain

$$
\begin{gathered}
\left(D^{2}-a^{2}\right)^{2} W-a^{2} R a \theta-\frac{R s}{L e} a^{2} \xi=0, \\
W+\left(D^{2}-a^{2}\right) \theta+D f\left(D^{2}-a^{2}\right) \xi=0, \\
W+\operatorname{Sr}\left(D^{2}-a^{2}\right) \theta+\frac{1}{L e}\left(D^{2}-a^{2}\right) \xi=0,
\end{gathered}
$$

where $a=\sqrt{a_{x}^{2}+a_{y}^{2}}$ and $D=\frac{d}{d z}$.

Following the proportional feedback control in [25], the continuously distributed actuators and sensors are arranged in a way that for every sensor, there is an actuator positioned directly beneath it. The determination of control, $q(t)$ can be accomplished using the proportional-integral-differential (PID) controller of the form:

$$
q(t)=r+K[e(t)] \text { where } e(t)=\hat{m}(t)+m(t)
$$

where $K=K_{p}+K_{D} \frac{d}{d t}+K_{l} \int_{0}^{t} d t$. Based on Equation (19), for one sensor plane and proportional feedback control, the actuator modifies the heated surface temperature using a proportional relationship between the upper, $\mathrm{z}=1$ and the lower, $\mathrm{z}=0$ thermal boundaries for perturbation field:

$$
T^{\prime}(x, y, 0, t)=-K T^{\prime}(x, y, 1, t) \text {. }
$$

Equations (16)-(18) are solved subject to the appropriate boundary conditions that are:

$$
W=D W=\theta(0)+K \theta(1)=\xi=0 \text { at } z=0
$$

For upper free boundary which is at $z=1$, we have

$$
W=D \theta=\xi=D^{2} W=0 \text { at } z=1
$$

For upper rigid boundary which is at $z=1$, we have

$$
W=D \theta=\xi=D W=0 \text { at } z=1
$$

The Galerkin-type weighted residuals method is applied to find an approximate solution to the system. The variables are written in a series of basis function as:

$$
W=\sum_{p=1}^{N} A_{p} W_{p}, \theta=\sum_{p=1}^{N} B_{p} \Theta_{p} \text { and } \xi=\sum_{p=1}^{N} C_{p} \Lambda_{p},
$$

where the trial functions and will be chosen respective to the boundary conditions and are constants. Substitute Equation (24) into Equation (16)-(18) and make the expressions on the left-hand sides of those equations (the residuals) orthogonal to the trial functions, thereby obtaining a system of $3 N$ linear algebraic equations in the $3 N$ unknowns. The vanishing of the determinant of coefficients produces the eigenvalue equation for the system. One can regard $R a$ as the eigenvalue, and thus $R a$ is found in terms of the other parameters.

Performing the integration by parts with respect to $z$ between $z=0$ and 1. By using the boundary conditions (21)(23), we obtain the system of linear homogeneous algebraic equations:

$$
\begin{aligned}
C A_{i}+D G_{i}+E D_{i} & =0, \\
F X_{i}+G A_{i}+H G_{i} & =0, \\
I D_{i}+J X_{i}+K A_{i} & =0,
\end{aligned}
$$

where

$$
\begin{aligned}
& C=\left\langle\left(D^{2} W\right)^{2}\right\rangle+2 a^{2}\left\langle(D W)^{2}\right\rangle+a^{4}\left\langle W^{2}\right\rangle \\
& D=a^{2}\langle W \Theta\rangle \\
& E=\frac{a^{2} R s}{L e}\langle W \Lambda\rangle \\
& F=\langle W \Theta\rangle, \\
& G=-(D \Theta)^{2}-a^{2}\left\langle\Theta^{2}\right\rangle, \\
& H=-D f\langle D \Lambda D \Theta\rangle-a^{2} D f\langle\Theta \Lambda\rangle, \\
& I=\langle W \Lambda\rangle, \\
& J=-S r\langle D \Theta D \Lambda\rangle-a^{2} S r\langle\Lambda \Theta\rangle, \\
& K=-\frac{1}{L e}\left\langle(D \Lambda)^{2}\right\rangle-\frac{a^{2}}{L e}\left\langle\Lambda^{2}\right\rangle
\end{aligned}
$$


where the angle bracket $\langle\cdots\rangle$ denotes the integration with respect to $z$ from 0 to 1 . The vanishing of coefficients produces the eigenvalue equation for the system.

\section{RESULTS AND DISCUSSION}

The linear stability analysis is carried out to investigate the influence of feedback control on the double-diffusive binary fluid layer. Therefore, Fig. 1-14 have been plotted for such objective. Various boundary conditions have been used for instant free-free, rigid-free and rigid-rigid referring to upper-lower boundaries. The sensitiveness of the critical Rayleigh number, $R a_{c}$ to the changes of the difference physical parameters $K, R s, D f$, and $S r$ are also studied.

To validate our analysis [34], we have compared our results with [14] in the absence of feedback control, $K$ as can be seen in Table I. We recover the critical Rayleigh number for all types of boundaries as previously obtained by [14] and we extend the results by scrutinizing the effects of $K$ in the presence of Dufour, $D f$, and Soret, $S r$ parameter. From the table, we found that the increasing of the feedback control, $K$ in the binary fluid mixture increases the critical Rayleigh number spontaneously in all types of boundaries considered. It is well understood that the onset of convection occurs when the Rayleigh number reaches some critical value. The higher the critical number, the slower the onset of convection occurs in one heated system. This finding agrees well with [25]-[31] that reported the use of feedback control strategy is capable of delaying the onset of convection in one system. Observing the critical Rayleigh values of different types of boundaries, it is interesting to take note that the critical value of rigid-rigid boundary is the highest compared to the other types of boundaries of all $K$ considered. This could suggest the use of rigid-rigid boundary as it can hold stability in the system.

TABLE I

COMPARISON OF CRITICAL RAYLEIGH NUMBERS WITH VARIOUS TYPE OF BOUNDARIES AND FEEDBACK CONTROL, $K$

\begin{tabular}{|l|l|l|l|l|}
\hline \multirow{2}{*}{$\begin{array}{l}\text { Lower- } \\
\text { Upper }\end{array}$} & \multicolumn{2}{|c|}{$\boldsymbol{K = 0}$} & $\boldsymbol{K}=\mathbf{3}$ & $\boldsymbol{K}=\mathbf{5}$ \\
\cline { 2 - 5 } & Nield (2011) & \multicolumn{3}{c|}{ Present } \\
\hline Free-free & 657.50 & 657.73 & 1358.82 & 1751.00 \\
\hline Rigid-free & 1140.0 & 1138.7 & 1710.88 & 2134.02 \\
\hline Rigid-rigid & 1750.0 & 1749.9 & 2932.44 & 3551.81 \\
\hline
\end{tabular}

The variation of Rayleigh number $(R a)$ with wavenumber (a) and various values of feedback control $(K)$ is shown in Fig. 1. We interested to check the respond of $R a$ in the presence of $K$, and we choose $K=3,6,9$ and set other parameters to be constant. Obviously, from the graph, the curves shifted upwards as the values of $K$ increase. The patterns appear consistently in all types of boundaries as can be seen clearly in the graph. This indicates that the use of feedback controller in the doubly diffusive system elevates the thermal convection values which are $R a$ numbers and thus leads to a more stable system. Scrutinizing the difference impact on lower boundaries, we found that the free-free system has low critical values making this type of boundary is the most unstable boundary of one system. Since in this type of system, the particles of the liquids carry the heat without any obstacles, which consequently results in the transfer of heat easily. As illustrated in the figure, the rigidrigid boundary is the most stable boundary condition compared to rigid-free and free-free boundaries.

Fig. 2 is a variation of Rayleigh number versus wavenumber (a) with a different effect of Solutal Rayleigh number. According to [15], the effective thermal conductivity is reduced through the influences of thermosolutal Rayleigh number, $R s$ on the system and thus stabilizes it. To describe this, we have chosen the values of solutal Rayleigh number, $R s=500,1000,1500$ and we observed a positive increment of Rayleigh number, $R a$ as we increase the values of solutal Rayleigh number in all type of boundaries considered.

The trends of stability curves for the effect of Soret and Dufour parameters within the system have been plotted in Fig. 3 and Fig. 4 respectively. Soret effect is the occurrence of a diffusion flux due to a temperature gradient. Since the system is heated from below, the increase in the temperature flux contributes to the initiation of natural convection in the binary fluid mixture. As can be seen clearly in the graph, an increase of the Soret parameter, decrease the values of Rayleigh number and thus enhance convection in the system. Contrast to the Soret effect, an increasing of Dufour parameter; $D f$ increases the critical Rayleigh number as illustrated in Fig. 4. This behavior is manifest due to the energy flux from lower to higher solute concentration driven by the mass gradient in the binary system. It is worth to take note that the influence of $D f$ significantly alters the $R a$ values in all lower-upper boundary considered. These findings agree well with those reported by [15]. The graphs also reveal that the rigid-rigid boundary is still the most stable condition where the Rayleigh number recorded in both figures is the highest.

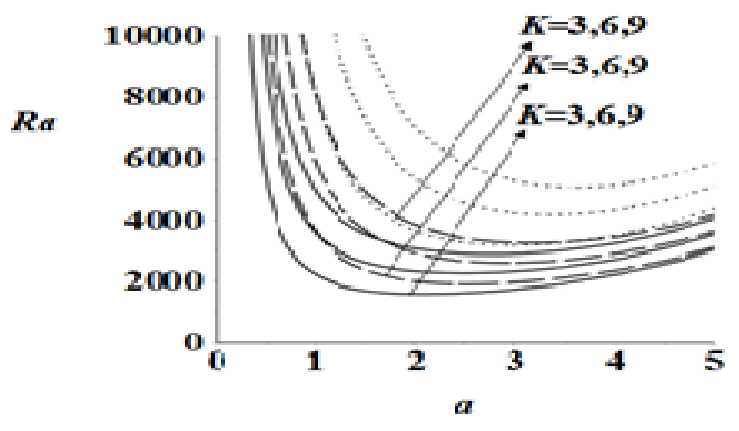

- Free-Free - - Rigid-Free …Rigid-Rigid Fig.1 The variation of $R a$ for different values of $K$

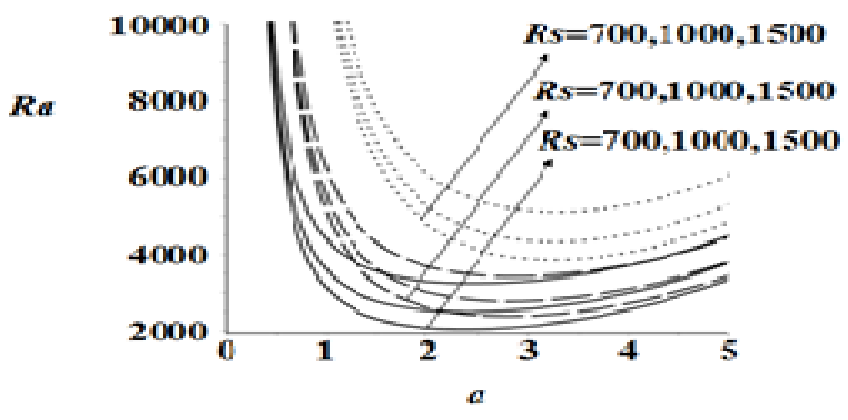

-Free-Free - - Rigid-Free ..... Rigid-Rigid Fig. 2 The variation of $R a$ for different values of $R s$ 


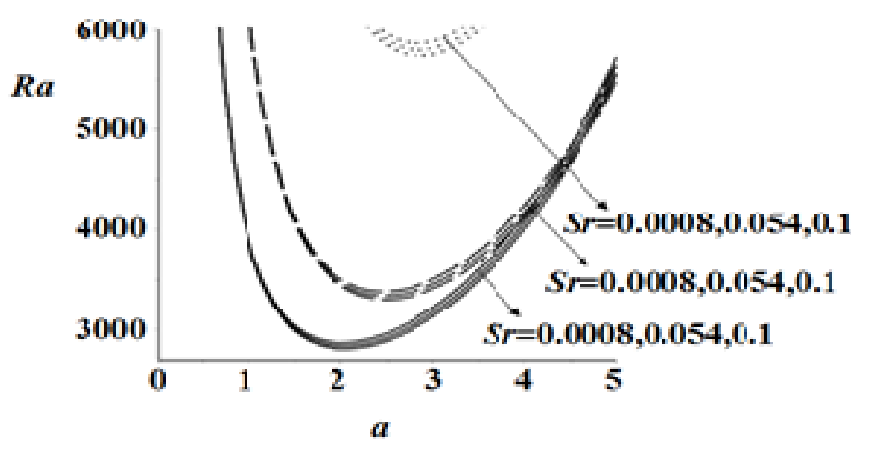

-Free-Free - - Rigid-Free -...-Rigid-Rigid

Fig. 3 The variation of $R a$ for different values of $S r$

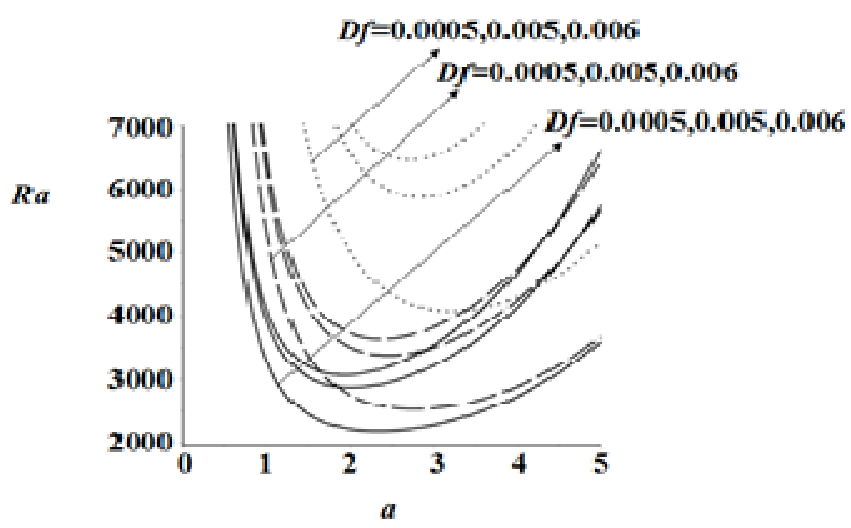

- Free-Free - - Rigid-Free $\cdots \cdot$ Rlgld-Rlgld

Fig. 4 The variation of $R a$ for different values of $D f$
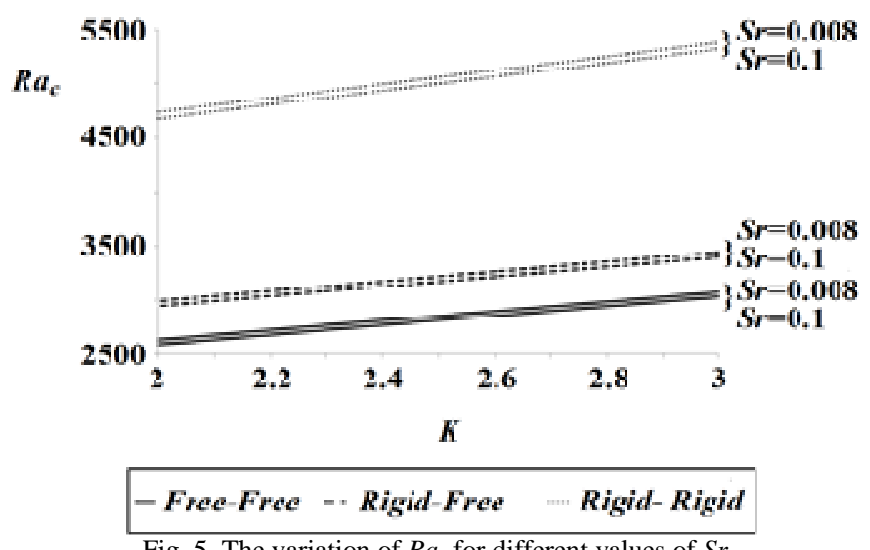

Fig. 5 The variation of $R a_{c}$ for different values of $S r$

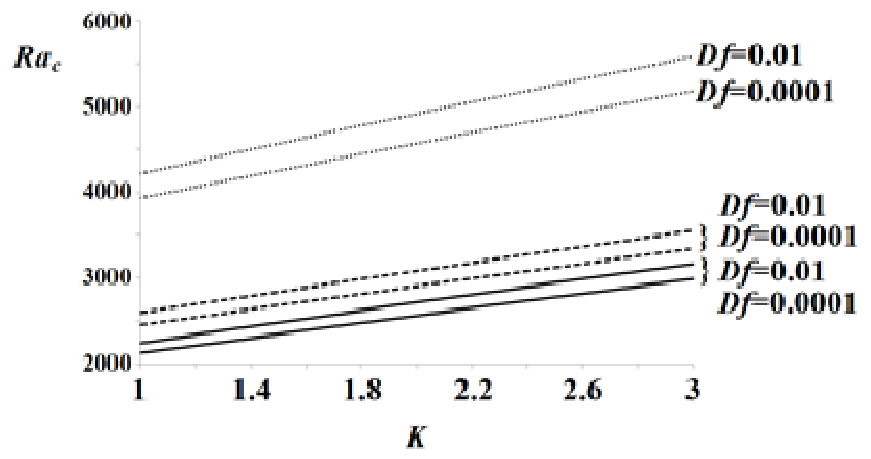

- Free-Free =: Rigid-Free $\quad$ Rigid-Rigid

Fig. 6 The variation of $R a_{c}$ for different values of $D f$
The onset of convection occurs beyond a critical value of the Rayleigh number. It is an interesting and crucial part of this investigation to check the effects of feedback control, Solutal Rayleigh number, Soret parameter and Dufour parameter on the onset of convection in a binary mixture. To reveal, we illustrated the findings in Fig. 5-9.

Fig. 5 and Fig. 6 are plotted in order to analyze the effect of feedback control, $K$ for variation values of $S r$ and $D f$ on the onset of natural convection in a binary mixture. In Fig. 5, it can be seen clearly that the onset of convection starts at a range of critical Rayleigh number from 2500 to 4700 at $K=$ 2, whereas the $R a_{c}$ recorded in Fig. 6 ranged from 2100 to 4200 when $K=1$. It is well known that the critical Rayleigh number in the Newtonian fluid layer is approximately 1700 for a closed surface and 1100 for a free surface as [32]. As observed, the critical Rayleigh number in Binary mixture is much larger compare to ordinary fluid layer, and this may be attributed to the fact that the Binary mixture of solute concentration packed in the system helps to slow down the initiation of convection. In the both figures, the critical Rayleigh number, $R a_{c}$ for the various values of $S r$ and $D f$ increases slightly with the increase of feedback controller, $K$. This reveals that an increasing of the feedback control, $K$ leads to a more stable system.

The critical Rayleigh number, $R a_{c}$ as a function of thermo-solutal Rayleigh number, Rs for different values of $K, S r$ and $D f$ are plotted in Fig. 7-9. In order to analyze the stability behavior of the system, we increase the values of solutal Rayleigh number, Rs for all bounded boundary considered. As expected, a large value of solutal Rayleigh number, $R s$ increases the critical Rayleigh number with various feedback control values, Soret and Dufour parameters. This implies that $R s$ yields a reduction in the rate of convection and stabilizes the system. As the impact on $\mathrm{Sr}$ and $D f$, an increasing of thermodiffusion in all of the figures remained the same which is to destabilize the mixture, and the reciprocal behavior can be found when increasing the energy flux that exhibits in the binary system [33].

It is noted that a positive trend exists on Fig. 9 when both effects of the thermo-solutal Rayleigh number $(R s)$ and feedback control $(K)$ are elevated, the critical Rayleigh number is increased.

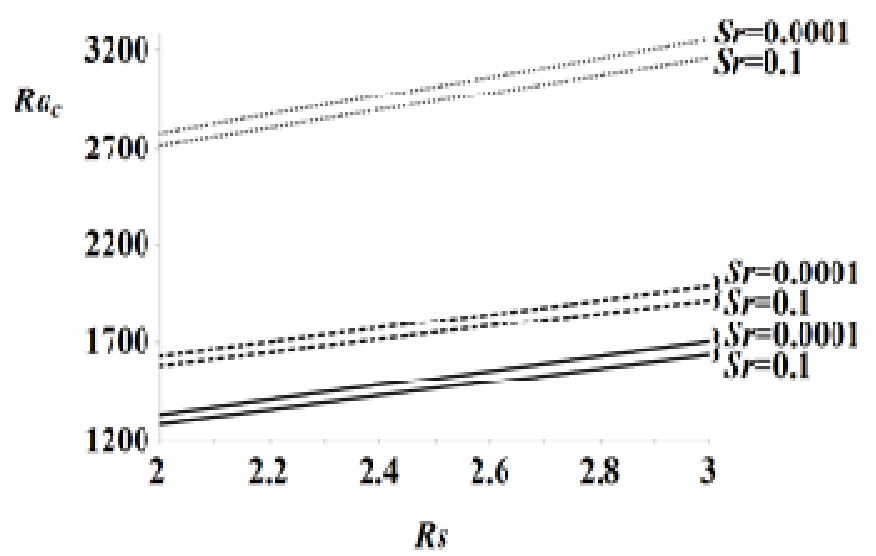

-Free-Free --Rigid-Free - Rigid-Righd

Fig. 7 The variation of $R a_{c}$ for different values of $\mathrm{Sr}$ 

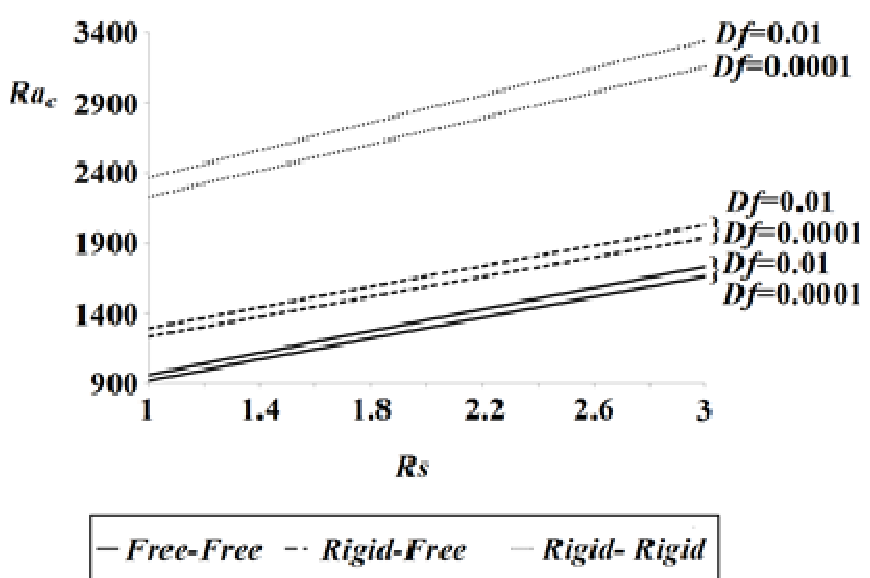

Fig. 8 The variation of $R a_{c}$ for different values of $D f$

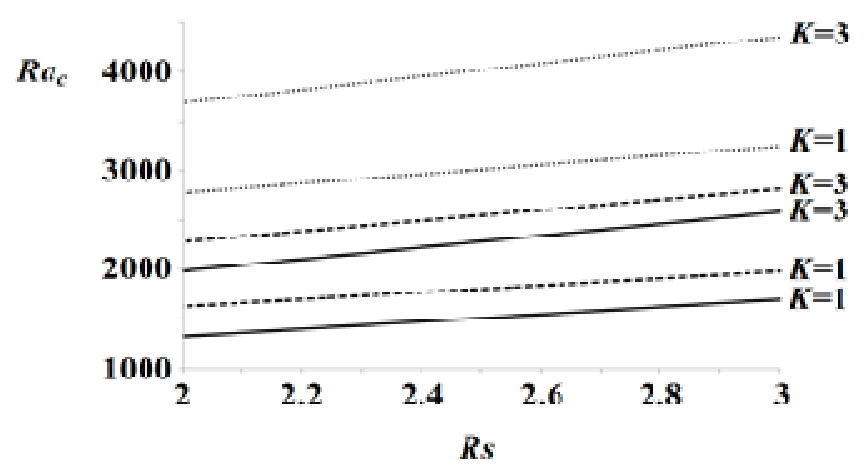

-Free-Free = Kigid-Free - Kigid-Rigid

Fig. 9 The variation of $R a_{c}$ for different values of $K$

It is certainly shown that the effect of feedback control, $K$ and Rs leads to stabilize the binary mixture system. As the effect of lower-upper boundary, the onset of convection happens rapidly in the free-free type of system, followed by the rigid-free and rigid-rigid system. This observation could point out that the use of rigid-rigid boundary can sustain stability in one system.

\section{CONCLUSION}

In the present paper, it has been demonstrated that the onset of double-diffusive convection in a horizontal binary fluid layer can be controlled. The problem has been investigated analytically using linear stability theory. Three types of boundary conditions on the horizontal boundaries that are free-free, rigid-free and rigid-rigid are considered in this investigation. The system is heated from below by a fix and constant heat flux. The influences of various parameters and feedback control on the onset of double-diffusive convection have been observed. Through the use of controller strategy, the performance of feedback control, $K$ in binary fluid has elevated the value of critical Rayleigh number monotonically. The controller delays loss of stability which means helps to reduce the intensity of RayleighBenard convection and thus sustain the stability of the system. For all cases considered in this study, the Dufour parameter, $D f$ has a stabilization effect and the Soret parameter; $S r$ drives a destabilization effect within the system. It is also noted that the influence of increasing the effect of $R s$ is to cause a delay on the onset of convection. Based on the results obtained, for the three types of boundary conditions chosen, free-free, rigid-free and rigidrigid, it is found that the system with rigid-rigid horizontal boundaries is the most stable followed by rigid-free and freefree horizontal boundaries.

\section{ACKNOWLEDGMENT}

Authors gratefully acknowledge the financial support received from Ministry of Higher Education Malaysia under Fundamental of Research Grant Scheme (02-01-151703FR).

\section{REFERENCES}

[1] H. Stommel, A.B. Arons, and D. Blanchard, "An oceanographical curiosity: The perpetual salt fountain," Deep Sea Research, vol. 3, pp. 152-153, Feb. 1956.

[2] M. E. Stern, "The salt fountain and thermohaline convection," Tellus, vol. 12, pp. 172-175, May 1960.

[3] D. A. Nield, "The thermohaline Rayleigh-Jeffreys problem," Journal of Fluid Mechanics, vol. 29, pp. 545-558, Sep. 1967.

[4] D. T. J. Hurle and E. Jakeman, "Soret driven thermosolutal convection," Journal of Fluid Mechanics, vol. 47, pp. 667-687, Jun. 1971.

[5] J. K. Platten and G. Chavepeyer, "Oscillatory motion in Benard cell due to the Soret effect," Journal of Fluid Mechanics, vol. 60, pp. 305-319, Sep. 1973.

[6] D. R. Caldwell, "Non-linear effects in a Rayleigh-Benard experiment," Journal of Fluid Mechanics, vol. 42, pp. 161-175, Jun. 1970.

[7] H. E. Huppert and J. S. Turner, "Double-diffusive convection," Journal of Fluid Mechanics, vol. 106, pp. 299-329, May 1981.

[8] J. K. Platten and J. C. Legros, Convection in Liquids, Heidelberg, Germany: Springer Berlin, 2012.

[9] A. Kadiri, H. B. Hamed, and H. Beji, "Linear stability analysis of mixed convection under soret effect in bounded horizontal fluid layer with slip conditions," American Journal of Mechanics and Applications, vol. 3, pp. 19-26, 2015.

[10] S. J. Linz, "Binary mixtures: Onset of Dufour driven convection," Physical Review A, vol. 40, pp. 7175-7181, Dec. 1989.

[11] A. Bergeon, D. Henry, H. Benahadid, and L. S. Tuckerman, "Marangoni convection in binary mixtures with soret effect," Journal of Fluid Mechanics, vol. 375, pp. 143-177, Nov. 1998.

[12] S. Slavtchev, G. Simeonov, S. V. Vaerenbergh, and J. C. Legros, "Marangoni instability of a layer of binary liquid in the presence of nonlinear Soret effect," International Journal of Heat and Mass Transfer, vol. 42, pp. 3007-3011, Aug. 1999.

[13] Y. H. Gangadharaiah, "Penetrative Marangoni convection in fluid layer with Soret effect," Journal of Advanced Mathematics and Applications, vol. 4, pp. 11-16, Jun. 2015.

[14] D. Yadav, D. Lee, H. H. Cho, and J. Lee, "The onset of doublediffusive nanofluid convection in a rotating porous medium layer with thermal conductivity and viscosity variation: A revised model," Journal of Porous Media, vol. 19, pp. 31-46, 2016.

[15] N. F. M. Mokhtar and J. Uddin, "Influence of internal heat source on double-diffusive soret induced convection in a binary fluid," in Proc. IEEE ICREM7'15, 2015, p. 88.e

[16] J. Tang and H. H. Bau, "Stabilization of the no-motion state in Rayleigh-Benard convection through the use of feedback control," Physical Review Letters, vol. 70, pp. 1795-1798, Mar. 1993a.

[17] J. Tang and H. H. Bau, "Feedback control stabilization of the nomotion state of a fluid confined in a horizontal porous layer heated from below," Journal of Fluid Mechanics, vol. 257, pp. 485-505, Dec. $1993 \mathrm{~b}$.

[18] J. Tang and H. H. Bau, "Stabilization of the no-motion state in the Rayleigh-Benard problem," Proceedings of the Royal Society London A, vol. 447, pp. 587-607, 1994.

[19] J. Tang and H. H. Bau, "Stabilization of the no-motion state of a horizontal fluid layer heated from below with Joule heating," ASME Journal of Heat Transfer, vol. 117, pp. 329-333, May 1995.

[20] Y. P. Hu, Y. R. Li, and C. M. Wu, "Aspect ratio dependence of Rayleigh-Bénard convection of cold water near its density maximum 
in vertical cylindrical containers," International Journal of Heat and Mass Transfer, vol. 97, pp. 932-942, Jun. 2016.

[21] D. N. Guzman, T. Frączek, C. Reetz, C. Sun, D. Lohse, and G. Ahlers, "Vapour-bubble nucleation and dynamics in turbulent Rayleigh-Bénard convection," Journal of Fluid Mechanics, vol. 795, pp. 60-95, May 2016.

[22] L. E. Howle, "Linear stability analysis of controlled Rayleigh-Benard convection using shadowgraphic measurement," Physics of Fluids, vol. 9, pp. 3111-3113, Nov. 1997.

[23] J. Tang and H. H. Bau, "Experiments on the stabilization of the nomotion state of a fluid layer heated from below and cooled from above," Journal of Fluid Mechanics, vol. 363, pp. 153-171, May 1998a.

[24] J. Tang and H. H. Bau, "Numerical investigation on the stabilization of the no-motion state of a fluid layer heated from below and cooled from above," Physics of Fluids, vol. 10, pp. 1597-1610, May 1998b.

[25] H. M. Azmi and R. Idris, "Control of Marangoni convection in a rotating fluid layer with cubic temperature gradients," in Proc. CMCGS'15, 2015, p. 97-103.

[26] N. Bachok, N. M. Arifin, and F. M. Ali, "Effects of control on the onset of Marangoni-Benard convection with uniform internal heat generation," Matematika, vol. 24, pp. 23-29, Jun. 2008.

[27] S. A. Kechil and I. Hashim, "Control of Marangoni instability in a layer of variable-viscosity fluid," International Communications in Heat and Mass Transfer, vol. 35, pp. 1368-1374, Dec. 2008.

[28] S. A. Kechil and I. Hashim, "Oscillatory Marangoni convection in variable-viscosity fluid layer: The effect of thermal feedback control," International Journal of Thermal Sciences, vol. 48, pp 1102-1107, Jun. 2009

[29] Z. Siri and I. Hashim "Control of oscillatory of Bénard-Marangoni convection in rotating fluid layer," in Proc. ICSPS'09, 2009, p. 938.

[30] N. H. Z. Abidin, N. F. M. Mokhtar, N. Arbin, J. M. Said, and N. M. Arifin, "Marangoni convection in a micropolar fluid with feedback control," in Proc. IEEE ISBEIA'12, 2012, p. 558.

[31] I. K. Khalid, N. F. M. Mokhtar, and N. M. Arifin, "Rayleigh-Benard convection in micropolar fluid with feedback control," World Applied Sciences Journal, vol. 2, pp. 112-118, 2013.

[32] O. Sano, Formation and Transitions of Patterns in Thermal Convection: Research of Pattern Formation, Japan: KTK Scientific Publishers, 1994.

[33] I. M. Yassin, A. Zabidi, M. S. A. M. Ali, N. M. Tahir, H. A. Hassan, H. Z. Abidin, and Z. I. Rizman, "Binary particle swarm optimization structure selection of nonlinear autoregressive moving average with exogenous inputs (NARMAX) model of a flexible robot arm," International Journal on Advanced Science, Engineering and Information Technology, vol. 6, pp. 630-637, Oct 2016.

[34] M. N. M. Nor, R. Jailani, N. M. Tahir, I. M. Yassin, Z. I. Rizman, and R. Hidayat, "EMG signals analysis of BF and RF muscles in autism spectrum disorder (ASD) during walking," International Journal on Advanced Science, Engineering and Information Technology, vol. 6, pp. 793-798, Oct. 2016. 